\title{
Maximum Power Point Tracking for a PV System Using Tuned Support Vector Regression by Particle Swarm Optimization
}

\author{
Ahmed G. Abo-Khali1* \\ Department of Electrical Engineering, College of Engineering, Majmaah University, Almajmaah 11952, Saudi Arabia \\ Department of Electrical Engineering, College of Engineering, Assiut University, Assiut 71515, Egypt \\ *Corresponding Author: a.abokhalil@mu.edu.sa
}

Submitted: $24 / 11 / 2019$

Revised: $02 / 02 / 2020$

Accepted: 17/02/2020

\begin{abstract}
The photovoltaic (PV) system is always operated at the maximum power point (MPP) condition irrespective of the fluctuations in PV voltage. The maximum power point tracking (MPPT) employed in PV system is not effective during the presence of current ripple as normal tracking becomes increasingly complex during fluctuation in solar irradiation or due to change in MPP condition. This paper proposes a high-efficiency power point tracking algorithm to minimize the current ripple and power oscillation around the maximum power point. The developed algorithm is based on particle swarm optimization-support vector regression (PSO-SVR) technique. The proposed algorithm is implemented to select and tune the Support Vector Regression (SVR) parameters such as kernel parameters, variance, and the penalty factor for predicting the irradiation level as well as to determine the PV voltage corresponding of maximum power point. The PSO method is used to accelerate the process of optimizing the SVR parameters at different conditions and get knowledge about the corresponding global optimum. From the experimental results, the efficiency of maximum power point tracking is found to be $99.8 \%$. The proposed algorithm PSO-SVR shows a better performance than using SVR alone. The stability and accuracy of MPPT have been validated during the rapid fluctuation of solar irradiation in the range of $25 \%$ to $100 \%$.
\end{abstract}

Keywords: PV MPPT; SVR; PSO; Grid-connected.

\section{Nomenclature}

$I_{p h}:$ the photocurrent;

$I_{d}:$ the diode saturation current;

Voc : open voltage;

$I_{s c}$ : short-circuit current $I_{s c}$;

$I_{o}$ : diode saturation current;

$k$ : Boltzmann constant;

$q$ : charge [C];

$R_{s h}$ : the parallel resistance;

$S_{N}$ : the unit solar irradiance; 
$B$ : the manufacturing constant;

$I_{t}$ : Short-circuit current temperature coefficient at surface temperature rise $[\mathrm{A} / \mathrm{K}]$;

$T_{c}$ : Solar cell temperature $[\mathrm{K}]$;

$T_{r}:$ Solar cell reference temperature $[\mathrm{K}]$;

$I_{o}$ : Reverse saturation current $[\mathrm{A}]$ at solar cell operating temperature;

$E_{g}$ : energy band gap (Si PN junction energy gap, $\left.1.12[\mathrm{eV}]\right)$.

\section{INTRODUCTION}

The importance of inducting Renewable Energy (RE) technologies based on Solar and Wind Energy resources is highly pertinent in the present environmental scenario of the world. The dependence on conventional energy resources such as coal and petroleum increases the risk of global warming and environmental challenges. The research and development on integrating RE resources into the energy mix of world countries are also active, and the most prominent and promising among them is solar energy (Awan A.B. et al., 2018, Abo-khalil A.G. et al., 2016, KAPSRC 2017).

The maximum power that can be extracted from a solar cell module varies due to external factors such as irradiation, load, and external temperature. Hence, it is essential to adopt proactive control techniques to minimize the energy loss so as to extract maximum power from the solar cell. MPPT control technique continuously operates the solar cell at maximum power point condition. Commonly used MPPT control methods include constant voltage control method, Perturbation and Observation (PO) method, Incremental Conduction (IncCond) method, fuzzy logic, neural network, and Newton method (Zell et al., 2015, Al-saleh et al., 2007, Abo-khalil et al., 2006, Yu et al., 2009).

In the constant voltage control method, the range of fluctuation for the output voltage of the solar cell array is small during the solar irradiation changes, and it exhibits almost constant voltage characteristics. In this method, control is relatively simple as it requires only voltage sensor and, hence, reduces the use of current sensors. However, the main disadvantage of this method is that during rapid fluctuations in solar irradiation, the maximum power point may not be found, thereby reducing the output efficiency (Karami et al., 2017)

In PO method, the maximum power point condition is obtained by continuously decreasing or increasing the output voltage of the photovoltaic cell. This control method is found to be relatively simple and has accurate tracking performance but suffers from a drawback that the oscillations near the MPP may fail to track the maximum power point according to external conditions (Elgendy et al., 2012, Femia et al., 2005, Mohanty et al., 2017).

The above-mentioned shortcoming of the PO method can be compensated in IncCond method. In this method, it is possible to follow the MPP condition since the derivative of the output power with respect to the solar cell voltage becomes '0' at the maximum power point (Safari et al., 2011, Hussein et al., 1995).

The MPPT method using fuzzy logic can express control rules easily based on expert knowledge and has strong characteristics against disturbance. In addition, when the control target cannot be accurately approximated mathematically, the MPP can be tracked through the input-output relationship of the control target. However, since the amount of computation is large, this method cannot be used on the CPU at low cost (Hohm et al., 2003, Spiazzi et al., 2009, Kottas et al., 2006, Farayola et al., 2017). Neural networks can obtain, store, and use information by learning. This learned information enables MPPT tracing. This method also increases the amount of computation, making it expensive to use on CPUs (Al-amoudi et al., 2000, Syafaruddin et al., 2009).

The Newton Method is one of the most efficient ways to find the approximate or actual value of a general function. But when the slope is small, the calculations are complicated, and subsequently it is difficult to arrive on the solutions (Lacerda et al., 2010, Abo-khalil 2011).

The searching-based MPPT methods are relatively easy to implement but as the constant duty ratio changes, a trade-off occurs between the steady state and the transient response. When the rate of change of duty ratio is set in 
accordance with steady-state stability, the transient response characteristic decreases, and the same when applied to consider transient response characteristic causes vibration in the steady-state (Veerachary et al., 2003, Piegari et al., 2010). In order to surpass the drawbacks of the searching-based methods, different Artificial Intelligent (AI) techniques are investigated in several studies. The SVR is found to be very resistant to the overlearning problem (Jian et al., 2007, Abo-khalil et al., 2008, Abo-khalil et al., 2012) as it employs the concept of minimizing upper bound of the generalization errors as compared to Artificial Neural Networks (ANNs), which aim at minimizing the training errors. SVR has been widely and successfully applied to many prediction and estimation problems. Since the SVR performance and accuracy depend on the choosing and tuning of the kernel and the penalty parameter, the parameter determination of SVR becomes a key problem. To optimize the selection of these parameters, Genetic algorithm (GA) or PSO can be used. However, for the sake of simplicity, high-optimization performance, and fast convergence, PSO has significant merits over the other optimization techniques.

A novel PSO-SVR algorithm is implemented in this paper to track the MPP of PV array. PSO is used offline using the training data so as to optimize the SVR parameters. The offline obtained parameters such as penalty factor, variance, and kernel parameters are tested online until the optimum values are obtained, which can be used to accurately extract the PV's MPP without power oscillations. The proposed algorithm is validated with the help of experimental results.

\section{PV MODEL}

The equivalent circuit of a solar cell using photovoltaic effect when light is irradiated is depicted in Fig.1. Parallel resistance $R_{s h}$ represents the leakage resistance represented by a constant resistance, which is beyond the ideal diode characteristics (Abo-khalil et al., 2019). Since the relationship between PV voltage and current is severely nonlinear, modeling of the PV must be preceded in order to perform a full system simulation that can achieve accurate system characteristics.

Ideally, short-circuit current is equal to the photocurrent, and the open cell voltage determined by the diode saturation current can be expressed by the following equation (Yu et al., 2009):

$$
V_{o c}=\frac{k T}{q} \ln \left[\frac{I_{p h}}{I_{d}}+1\right]
$$

Also, the relation between short-circuit current and open-voltage according to temperature change is as follows:

$$
I_{s c}=I_{o}\left[\exp \left(\frac{q V_{o c}}{k T}\right)\right]
$$

The current-voltage (I-V) and power-current (P-V) characteristics of the solar cell are expressed as follows (Hsiao et al., 2010):

$$
\begin{aligned}
& I_{p h}=I_{s c} S_{N}+I_{t}\left(T_{c}-T_{r}\right) \\
& I_{d}=I_{o}\left[\exp \left(\frac{q\left(V_{L}+I_{L} R_{s}\right)}{A k T}\right)-1\right] \\
& I_{o}=I_{o r}\left[\frac{T_{c}}{T_{r}}\right] \cdot \exp \left(\frac{q E_{g}}{B k}\left(\frac{1}{T_{r}}-\frac{1}{T_{c}}\right)\right) \\
& I_{L}=I_{p h}-I_{d}-\frac{V_{L}+I_{L} R_{s}}{R_{s h}}
\end{aligned}
$$


Equation (3) is valid for certain irradiation level $S_{N}$ and at a particular operating cell temperature $T_{c}$. The PV characteristics can be obtained by measuring the variation of the PV current, voltage, and power at different irradiation level and cell temperature. The I-V and P-V characteristics for various irradiation level and constant cell temperature are shown in Fig. 2.

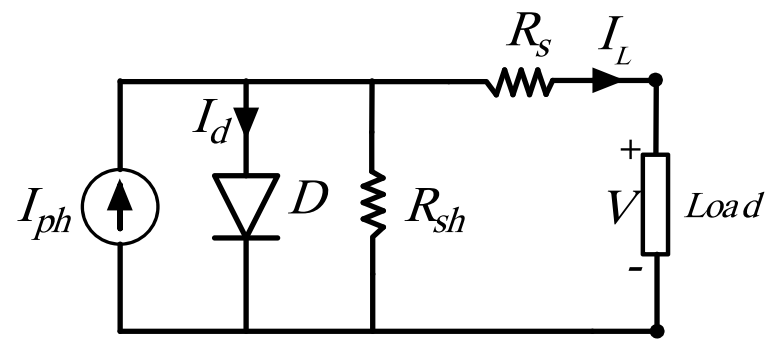

Figure 1. Equivalent circuit of PV array.
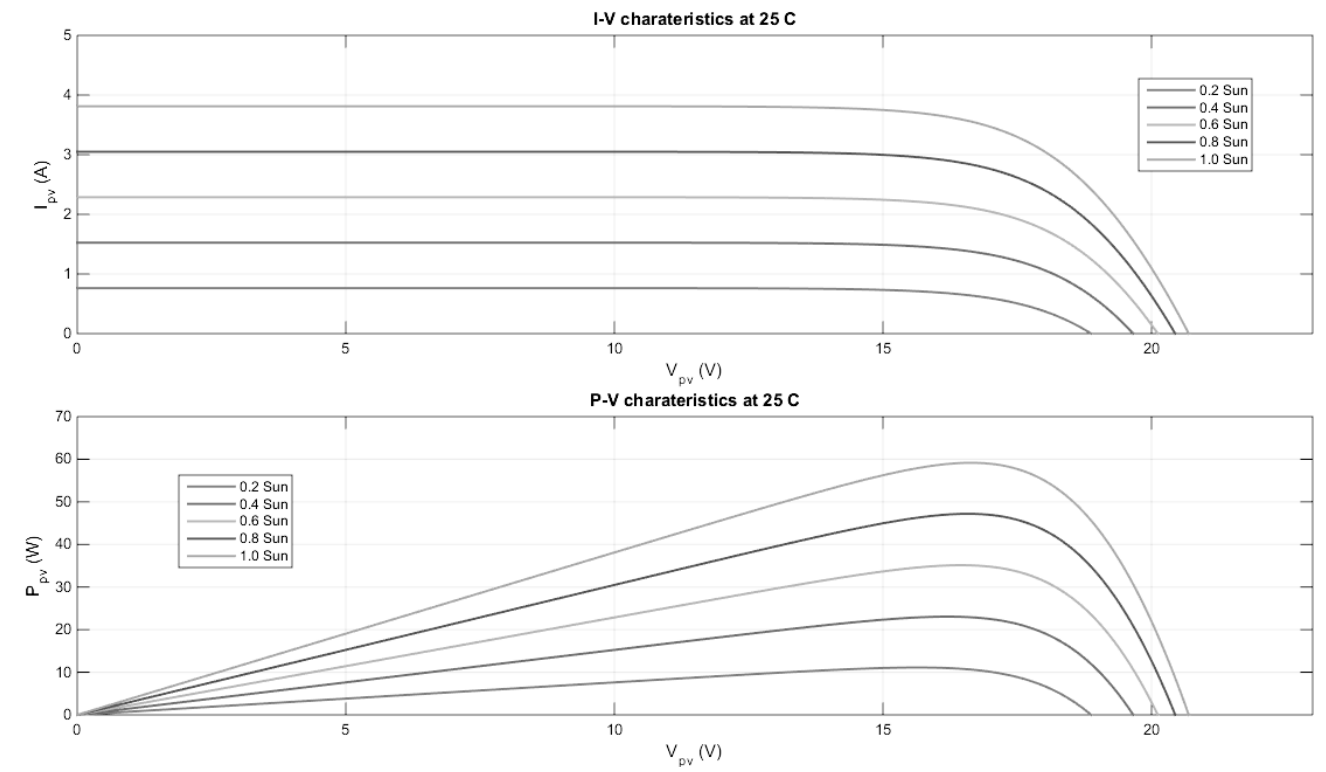

Figure 2. V-I and P-I characteristics at constant temperature.

\section{MPPT USING PSO-SVR METHOD}

Support Vector Machines (SVM) are a new kind of neural network algorithms proposed by Vapnik (Cortes et al., 1995, Vapnic et al., 1999, Muller et al., 1997). SVM has shown excellent prediction performance when applied to classification problem based on statistical learning theory. SVM is much easier to generalize as it is based on Structural Risk Minimization (SRM) than when compared to neural networks, which is based on empirical risk minimization (ERM) (Cherkassky et al., 1998). For this reason, SVM has been successfully implemented in various fields such as prediction, classification, and regression. Support Vector Regression (SVR) has been extended to regression problems by introducing an insensitive loss function to SVM regression models, while SVM has been applied to the prediction of classification problems (Abo-khalil et al., 2008). The SVM provides an optimal separation plane (hyperplane) to solve the classification problem. The reasons why SVM attracts attention is as follows. First, it is easy to interpret the results because it is based on a clear rationale. Second, it can achieve high level of artificial neural network in practical application.

The choice of a subset of functions is an important issue when creating a regression analysis system to construct a regression model based on SVM. The use of a small subset and appropriate characteristics can facilitate the 
implementation of regression decisions. In addition to feature selection, the appropriate model parameters can improve the accuracy of RVS prediction. To design the SVR algorithm, the kernel parameters must be set to determine the soft margin $C$. The parameters to be optimized include parameters of the kernel function, such as the penalty parameter $C$ and sigma for the function radial base (RPF) kernel.

Therefore, the proper selection of subsets of functions and the parameter settings of the model have a significant impact on the regression accuracy. The selection of the characteristic subsets affects the appropriate parameters of the kernel, so it is better to obtain the subset of features and the SVR parameters at the same time. There are several optimization methods to find SVR parameters such as GA and PSO, and the latter is promising as discussed in the next sections.

\section{A. Support Vector Regression (SVR)}

As mentioned earlier, SVR algorithm can be used to estimate the unknown function between the system input and output from the training data. It is used to predict the output from the system input value online when the input/output relationship of the system is estimated correctly offline. The input and output data for offline training are given as $\left(x_{1}\right.$, $\left.y_{1}\right),\left(x_{2}, y_{2}\right), \ldots . .\left(x_{n}, y_{n}\right)$ where $x_{i}$ and $y_{i}$ are input and output vectors, and $n$ is the number of training data. The general function of SVR is as follows (Abo-khalil et al. 2007):

$$
f(x)=(w . \Phi(x))+b
$$

Here, $w$ represents the weight vector, $\underline{\mathrm{b}}$ is the bias, $\Phi$ is the nonlinear function, and is the vector dot product. The input data for the SVR consists of two variables, which are the PV voltage and power, while the output is the irradiation level and the PV voltage at maximum power. For ten different irradiation levels, twenty samples have been used in the training stage with a total number of two hundred points. To reduce the empirical risk in the SVR model, the slack variables are applied as in Eltamaly et al. (2020):

$$
R_{\text {reg }}(f)=\frac{1}{2}\|w\|^{2}+C \sum_{i=1}^{n} \Gamma\left(f\left(x_{i}\right)-y_{i}\right)
$$

where $\Gamma($.$) is the cost function, \varepsilon$ is the variance, and $\mathrm{C}$ is the penalty factor, which determines how much variation is allowed for the training data, and the complexity term of the model $\left(\|w\|^{2}\right)$. Slack variable $\left(\xi\right.$ and $\left.\xi^{*}\right)$ is introduced so that estimation problem can be determined even if training data exists.

The optimization solution is given by

$$
f(x)=\left(\sum_{i=1}^{n}\left(\alpha_{i}-\alpha_{i}^{*}\right) \cdot\left(\phi\left(x_{i}\right) \cdot \phi(x)\right)+b\right.
$$

subject to $0 \leq \alpha_{i} \leq C, 0 \leq \alpha_{i}^{*} \leq C$

Dual multipliers are used to solve the regression problem given in (13) using

$$
f(x)=\sum_{i=1}^{n}\left(\alpha_{i}-\alpha_{i}^{*}\right) \cdot K\left(x_{i}, x\right)+b
$$

where $K\left(x_{i}, x_{j}\right)=\Phi\left(x_{i}\right)^{T} \Phi\left(x_{j}\right)$ is the kernel function used for function approximation. As we have seen so far, SVR uses kernel functions to transform learning data into feature space points and then learn in feature space, but if SVM is a machine learning classifier, the difference is that it is a generalized method for predicting arbitrary real values using a regression function. The bias term $b$ in Eq. (15) can be calculated as follows:

$$
b=\text { mean }\left(\sum_{i=1}^{n} y_{i}-\left(\alpha_{i}-\alpha_{i}^{*}\right) \cdot K\left(x_{i}, x\right)\right)
$$

The radial base function $(\mathrm{RBF})$ is selected to solve the problem as follows: 


$$
K\left(x_{i}, x\right)=\exp \left\{-\frac{\left|x_{i}-x\right|^{2}}{\sigma^{2}}\right\}
$$

The regression model is obtained based on the offline training stage, which uses the previously collected data, and the learnt SVR model is subsequently fixed during the online estimation phase. The offline process is carried out by using MATLAB software. The long-time model training is prepared to be done in the offline phase. The training process produces the regression model with the calculated $\varepsilon, b$ and $C$. The next phase is the online calculation phase, the parameters are read in, and the model is built rapidly, then the measured values, which are the PV voltage and power, can be used into the model to quickly get the irradiation level and the optimum PV voltage. However, the determination of the optimum PV voltage is very sensitive to the model parameters. A high-optimization performance with a fast convergence should be used. PSO has significant merits in optimizing the SVR parameters as discussed in the following section.

\section{B. Particle Swarm Optimization}

Particle Swarm Optimization (PSO) algorithm is one of the optimization techniques that is inspired by the social behavior of animals and was proposed by Kennedy et al. (1995). Just as bio populations such as ants and bees share information about each individual's experience to find food and travel to the optimal location, the PSO algorithm makes it possible to find the optimal solution by balancing each other's variables. The PSO algorithm has the disadvantage of being prone to local minimum, but unlike other search algorithms, it can provide global optimization for large and complex functions like evolutionary operations and perform better than evolutionary operations. Due to its high speed, it has recently been attracting much attention and has been applied to various fields that are difficult to solve with existing algorithms. The PSO algorithm represents individual potential solutions as particles, and the number is initially determined by the dimension setting. The initialization and updates of PSO are randomly done in each calculation step using the standard PSO. An improvement for the random behavior of standard PSO is obtained by initializing the swarm based on the opposite numbers. A comparison between the position and velocity components using the standard PSO and the opposition PSO is then done to select the optimum position and velocity.

The $i^{t h} \mathrm{~d}$-dimensional particle is characterized by its location vector $P_{i}^{t}=P_{i 1}^{t}, P_{i 2}^{t}, \ldots, P_{i d}^{t}$ and its speed vector $v_{i}^{t}=v_{i 1}^{t}$, $v_{i 2}^{t}, \ldots, v_{i d}^{t}$. Each particle knows its best personal location $P_{i, B e s t}^{t}$ and the entire population's best global solution $G_{B e s t}^{t}$. The position of the population at iteration $\mathrm{t}$ is $P^{t}=P_{1}^{t}, P_{2}^{t}, \ldots, P_{N}^{t}$, where $N$ is the population size. Each particle updates its location according to (17) and (18):

$$
\begin{aligned}
& v_{i}^{t+1}=w^{t} v_{i}^{t}+c_{1} r_{1}\left(p_{i, B e s t}^{t}-p_{i}^{t}\right)+c_{2} r_{2}\left(p_{i, B e s t}^{t}-p_{i}^{t}\right) \\
& p_{i}^{t+1}=p_{i}^{t}+v_{i}^{t+1}, i=1,2,3, \ldots, N
\end{aligned}
$$

The constriction coefficients introduced in Lin et al. (2008) are used to set $c_{1}$ and $c_{2}$ :

$$
\begin{gathered}
x=\frac{2}{\phi-1+\sqrt{\phi^{2}-4 \phi}} \\
c_{1}=x \phi_{1}, c_{2}=x \phi_{2}
\end{gathered}
$$

where $\phi=\phi_{1}+\phi_{2}>4$. The optimization process is terminated when the best global solution results in a permissible fitness using the opposite PSO.

The SVR parameters are tuned by using PSO method as follows:

- enter original data for estimation, known as the data preparation step;

- $\quad$ initialize the particles with random velocities and positions, known as particles optimization; 
- $\quad$ perform an offline training process for SVR with training samples assessing each particle fitness value of the PSO for the SVR;

- $\quad$ update each particle velocity and position continuously to satisfy the termination condition; and

- construct and retrain the SVR estimation model based on the optimal parameters. The process of irradiation and maximum power point tracking estimation is shown in Fig. 3, which is described as follows.

1) Collect training data $\left(x_{i}, y_{i}\right)$ and determine the parameters $\varepsilon, C$ and $\sigma$ using PSO.

2) Determine kernel function $K\left(x_{i}, y_{i}\right)$ from the collected data.

3) Compute the Lagrange multipliers, $\mathrm{b}$, and $\alpha_{i}$ by minimizing the quadratic function in

$$
\begin{aligned}
& W\left(\alpha_{i}, \alpha_{i}^{*}\right)=\frac{1}{2} \sum_{i, j=1}^{n}\left(\alpha_{i}-\alpha_{i}^{*}\right)\left(\alpha_{j}-\alpha_{j}^{*}\right) K\left(x_{i}, x_{j}\right) \\
& -\sum_{i=1}^{n} y_{i}\left(\alpha_{i}-\alpha_{i}^{*}\right)+\frac{1}{2 C} \sum_{i=1}^{n}\left(\alpha_{i}^{2}-\alpha_{i}^{* 2}\right)
\end{aligned}
$$

subject to

$$
\sum_{i=1}^{n}\left(\alpha_{i}-\alpha_{i}^{*}\right)=0, \alpha_{i}, \alpha_{i}^{*} \in[0, C]
$$

$$
b=\operatorname{mean}\left(\sum_{i=1}^{n}\left\{y_{i}-\left(\alpha_{i}-\alpha_{i}^{*}\right) K\left(x_{i}, x_{j}\right)\right\}\right)
$$

The penalty factor in (21) is set to trade-off between learning errors and model complexity, and the slack variables are to allow errors in the training data.

The parameters in (22) can be computed offline using (16) and (21). According to the PSO, the optimum SVR parameters for PV MPPT are $\varepsilon=0.0024, \sigma=0.17$, and $C=284$.

Calculate the output of the estimator using (14) and (22) for any input $X$.

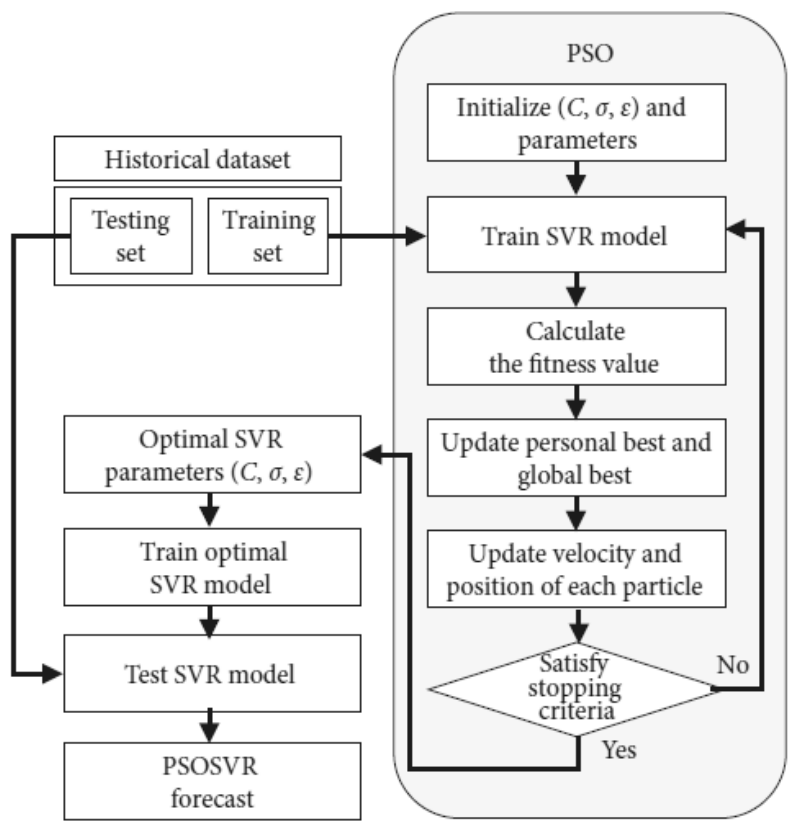

Figure 3. Flowchart for irradiation estimation and maximum power point tracking using PSO-SVR. 


\section{SYSTEM DESCRIPTION}

The PV power circuit is composed of a PV array, boost converter, DC-link capacitor, DC/AC inverter, and inductor filter, as shown in Fig. 4. In the grid-connected inverter, DC link voltage control and output stage current control are performed. The controller of the conventional boost converter is controlled so as to follow the voltage reference signal $\left(\mathrm{v}_{\mathrm{pv}}{ }^{*}\right)$ generated as a result of the MPPT algorithm. The switch is controlled by a gating signal of a current controller as shown in Fig. 5(a).

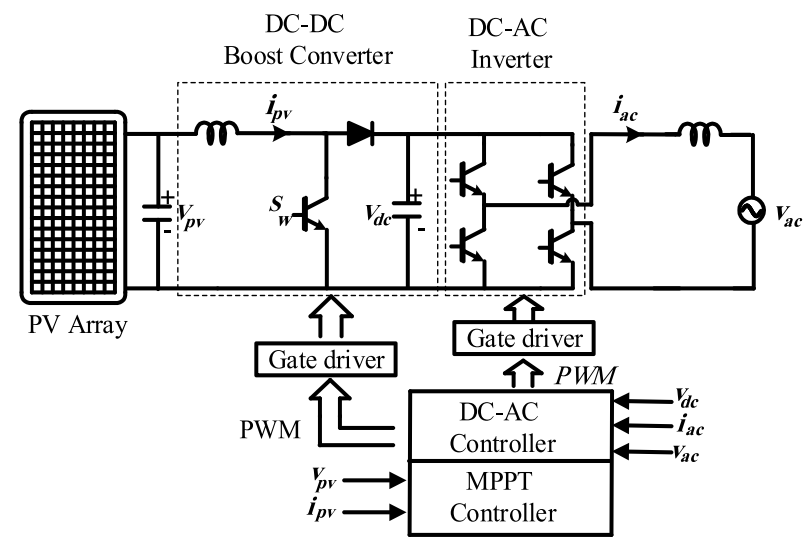

Figure 4. PV grid-connected system.

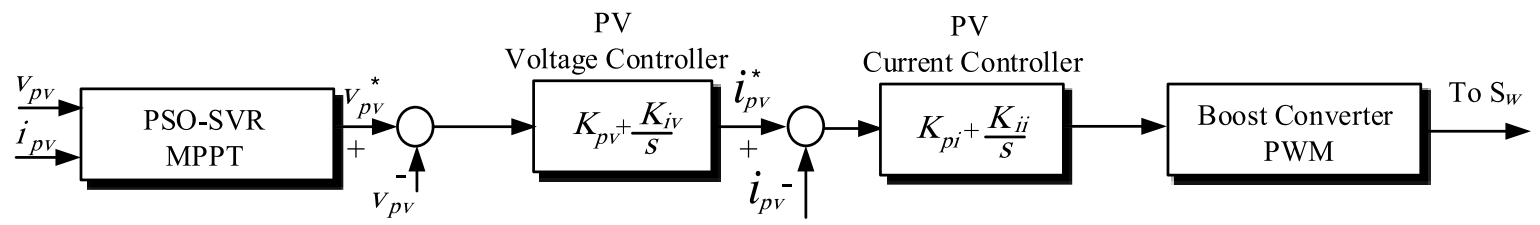

(a)

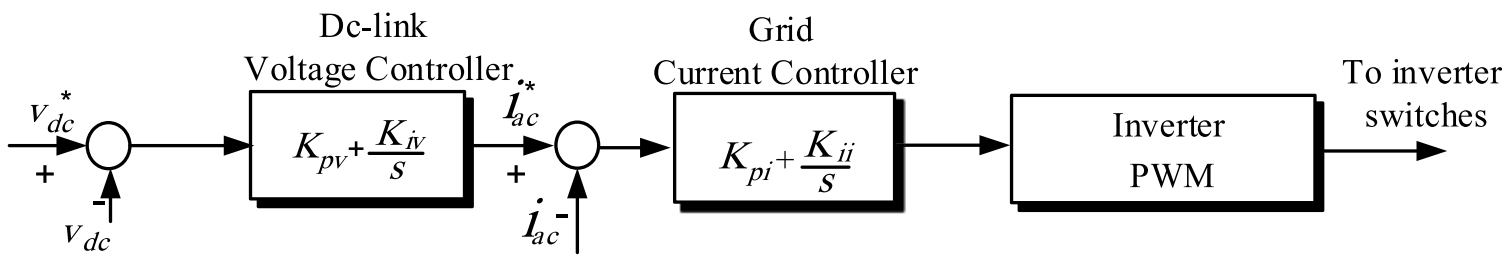

(b)

Figure 5. Control circuit for the boost converter.

The inverter, which converts DC to AC current that flows into the utility or local loads, also synchronizes the output current with the grid voltage and controls the dc link voltage. Figure 6 (b) shows the block diagram to control the inverter that includes the inner loop for current control and the outer loop for voltage control. One of the important functions of the inverter is to synchronize the output current with the grid voltage so as to maintain a unity power and control the dc-link voltage.

\section{EXPERIMENTAL RESULTS}

For validating the performance of the proposed PSO-SVR MPPT algorithm, an experimental setup is implemented using PV arrays, in which the specification is described in Table 1, DC-DC converter, inverter circuit, voltage, and current sensors. The system parameters are listed in Tables 1 and 2. 
Table 1. Parameters of the $60-\mathrm{W}$ Array at $25^{\circ} \mathrm{C}$ and $1000 \mathrm{~W} / \mathrm{m}^{2}$.

\begin{tabular}{|c|c|}
\hline Maximum power & $60 \mathrm{~W}$ \\
\hline MPP voltage $\left(V_{m p}\right)$ & $17.2 \mathrm{~V}$ \\
\hline MPP current $\left(I_{m p}\right)$ & $3.5 \mathrm{~A}$ \\
\hline Open-circuit Voltage $\left(V_{o c}\right)$ & $21.5 \mathrm{~V}$ \\
\hline Short-circuit current $\left(I_{s c}\right)$ & $3.85 \mathrm{~A}$ \\
\hline
\end{tabular}

Table 2. System Parameters.

\begin{tabular}{|l|l|}
\hline Grid voltage & $220 \mathrm{~V}$ \\
\hline Grid frequency & $60 \mathrm{~Hz}$ \\
\hline Filter inductance & $3 \mathrm{mH}$ \\
\hline DC-link Capacitor & $330 \mathrm{uF}$ \\
\hline
\end{tabular}

The performance of the proposed MPPT algorithm when the irradiation level changes from $500 \mathrm{w} / \mathrm{m}^{2}$ to $1000 \mathrm{w} /$ $\mathrm{m}^{2}$ is shown in Fig. 6 . The PV power is controlled to be at the MPP, which is $32 \mathrm{~W}$ and $60 \mathrm{~W}$, respectively, as shown in Fig. 6 (a). The PV voltage and current, in Fig. 6(b) and (c), are well regulated to extract the maximum with minimum low-frequency ripples. The grid current increases smoothly when the PV current increases as shown in Fig. 6(d). The grid voltage remains constant in all levels as shown in Fig. 6 (e).

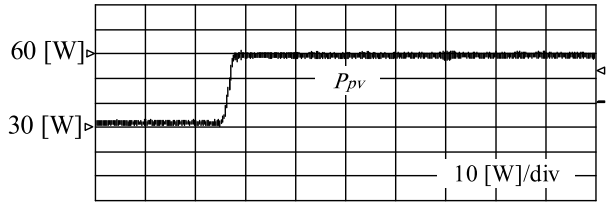

(a)

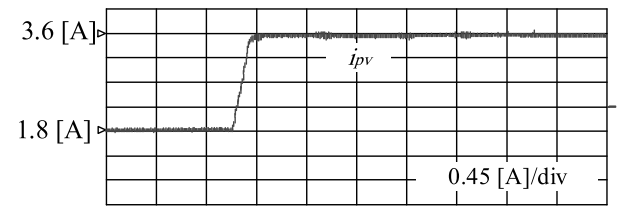

(b)

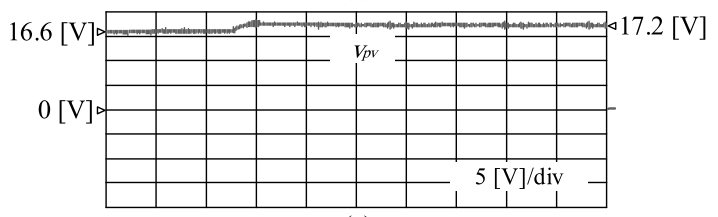

(c)

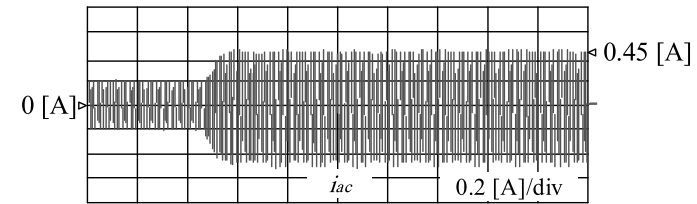

(d)

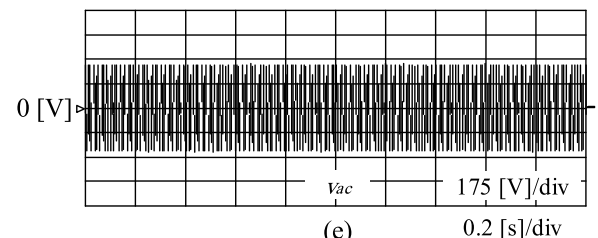

(e)

Figure 6. Proposed MPPT when irradiation increase $\left(500 \mathrm{~W} / \mathrm{m}^{2} \rightarrow 1000 \mathrm{~W} / \mathrm{m}^{2}\right)$. 
Figure 7 shows the PV power, current, and voltage when the PO MPPT method is used. It is obvious that the poor transient and the high oscillations are in steady state. It takes longer to reach steady state as shown in Fig. 7 (a), (b), and (c). From Fig. 6 and 7, it was found that the proposed method is 40ms when the irradiation level changes from $50 \%$ to $100 \%$, which is an advantage over the PO method. Based on the experimental results, the average value of the PV output is $59.91 \mathrm{~W}$, which is $99.85 \%$ of the 60 Watt when the OPSO-SVR method is used, while the PV average output power is 59.81 , which is $99.72 \%$ of the rated output power.

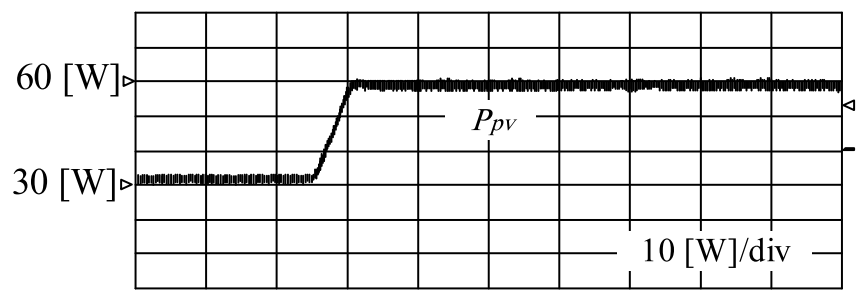

(a)

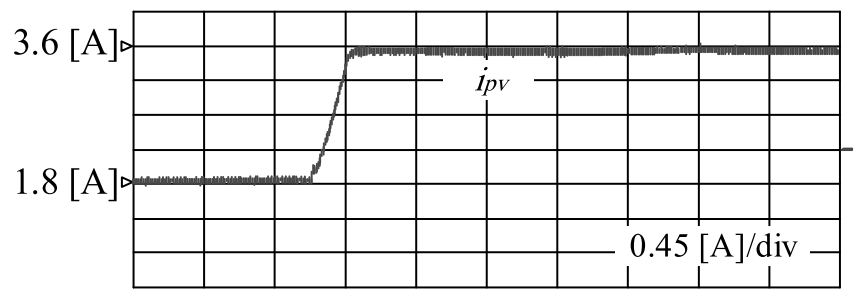

(b)

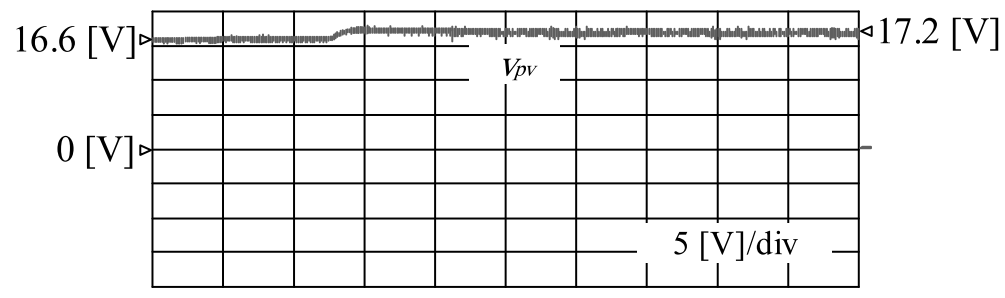

(c)

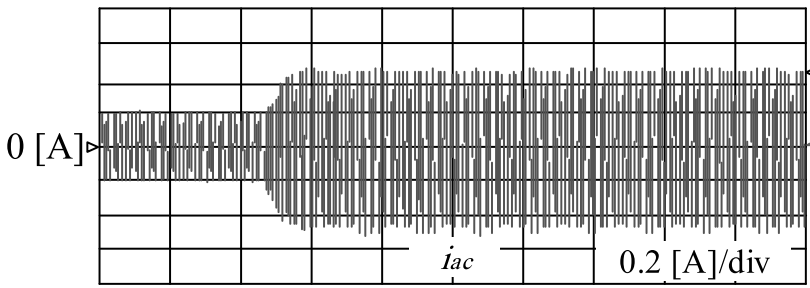

(d)

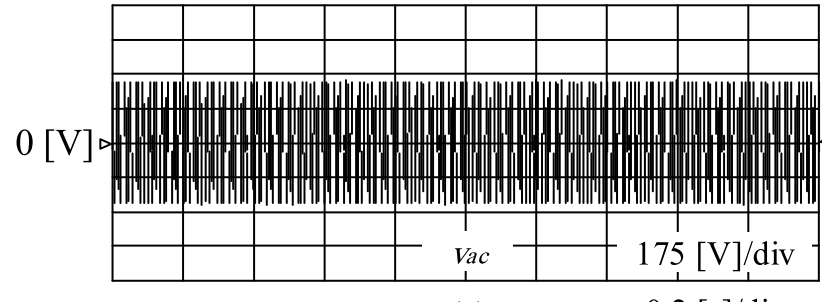

(e)

$0.2[\mathrm{~s}] / \mathrm{div}$

Figure 7. PO MPPT when irradiation increase $\left(500 \mathrm{~W} / \mathrm{m}^{2} \rightarrow 1000 \mathrm{~W} / \mathrm{m}^{2}\right)$. 
Figure 8 shows the steady-state and dynamic MPPT waveforms when the irradiation level changes from $1000 \mathrm{w} /$ $\mathrm{m}^{2}$ to $500 \mathrm{w} / \mathrm{m}^{2}$. The PV voltage is regulated to maintain the MPP with low oscillations around the maximum point.

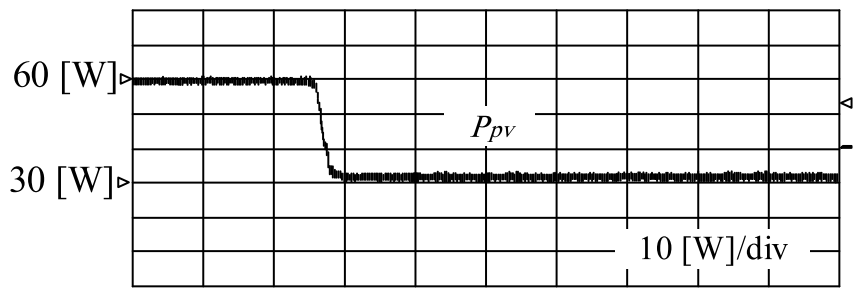

(a)

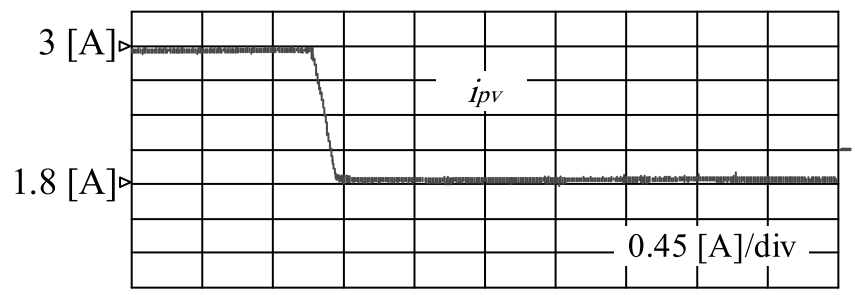

(b)

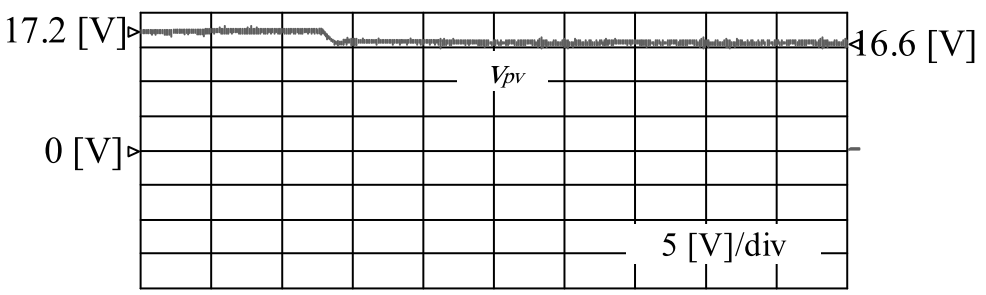

(c)

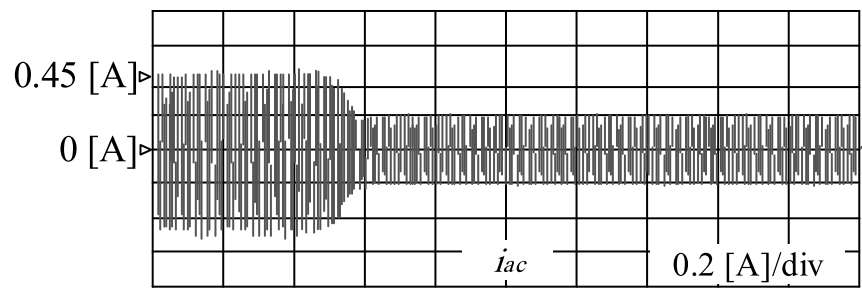

(d)

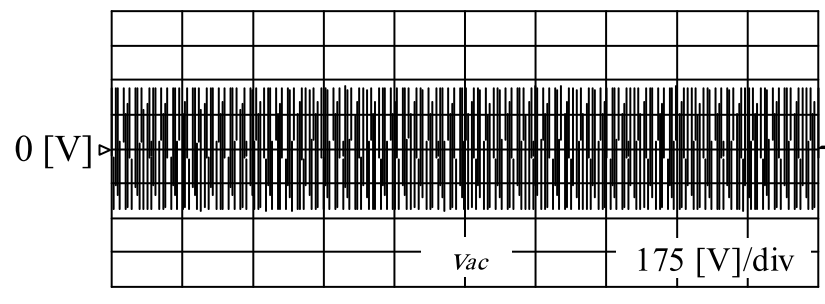

(e)

$0.2[\mathrm{~s}] / \mathrm{div}$

Figure 8. Proposed MPPT when irradiation decrease $\left(1000 \mathrm{~W} / \mathrm{m}^{2} \rightarrow 500 \mathrm{~W} / \mathrm{m}^{2}\right)$.

After calculating the input and output power in Fig. 8 (a), (d), and (e), it was found that the proposed MPPT efficiency is about $99.85 \%$, while the PO efficiency is about $99.72 \%$.

MPPT trajectories of both $P-V$ and $I-V$ curves are detailed in Fig. 9. The PV voltage ripple around the MPP at the 
steady state condition is found to be less than $0.2 \mathrm{~V}$ at $500 \mathrm{~W} / \mathrm{m}^{2}$ and $0.25 \mathrm{~V}$ at $1000 \mathrm{~W} / \mathrm{m}^{2}$. From the results, it is clear that the small variation in PV voltage ripple can ensure an MPPT efficiency greater than $99.8 \%$.

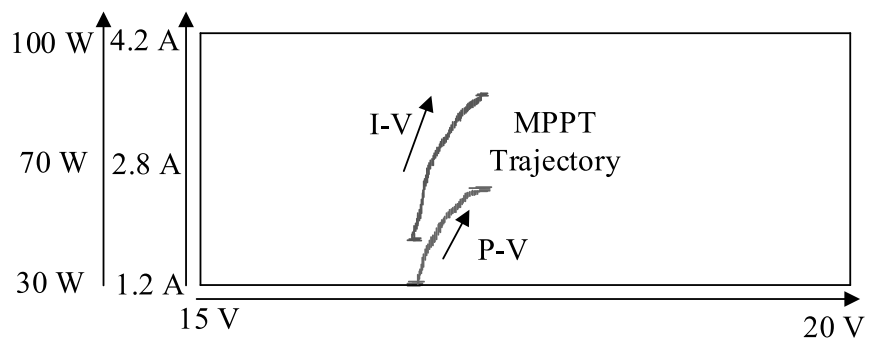

Figure 9. MPPT trajectories.

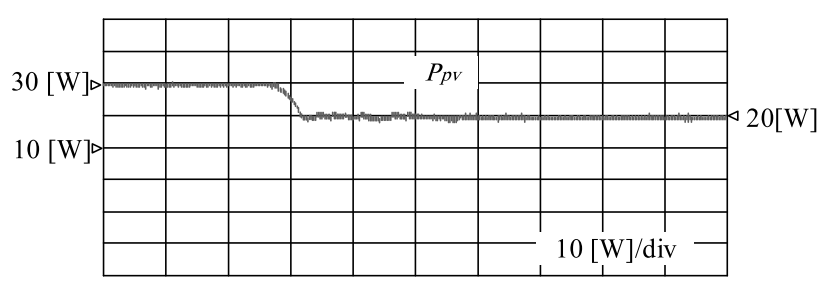

(a)

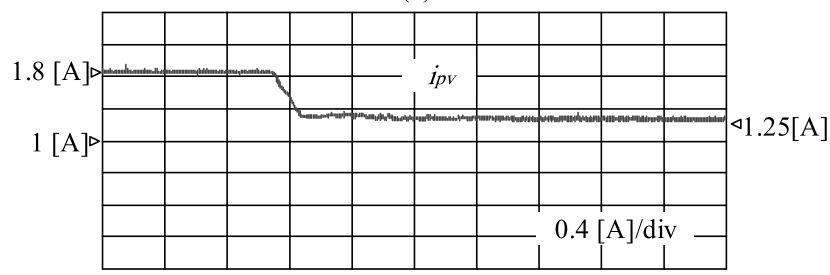

(b)

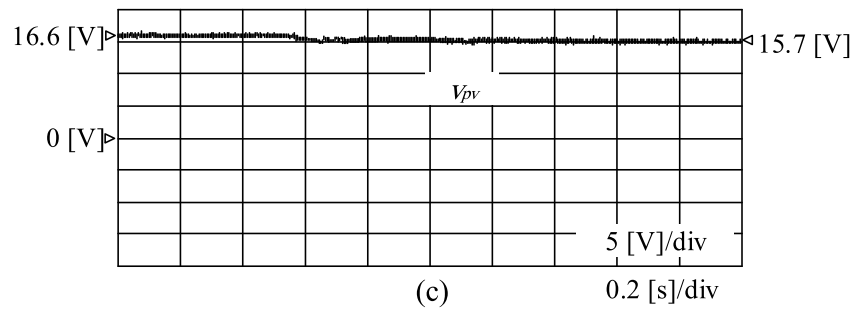

Figure 10. Proposed MPPT when irradiation decreases $\left(500 \mathrm{~W} / \mathrm{m}^{2} \rightarrow 250 \mathrm{~W} / \mathrm{m}^{2}\right)$.

Figure 10 shows the response characteristics of the PV of the proposed MPPT performance when the irradiation level changes from $500 \mathrm{~W} / \mathrm{m}^{2}$ to $250 \mathrm{~W} / \mathrm{m}^{2}$. Figure 10 (a) shows the output power of the PV module according to MPPT control method. The nominal power when the irradiation level is $500 \mathrm{~W} / \mathrm{m}^{2}$ is $30 \mathrm{~W}$, while the measured value is about 29.94 , which is about $99.79 \%$ of the nominal value. When the irradiation level changes to $250 \mathrm{~W} / \mathrm{m}^{2}$, the nominal output power is $20 \mathrm{~W}$, while the measured value is about 19.93 , which is about $99.68 \%$. This means that the tracking efficiency is reduced when the irradiation level is low. Figures 10(b) and (c) show the voltage and current change due to the irradiance change.

\section{CONCLUSION}

An MPPT controller meant for two-stage PV grid-connected system is implemented in this paper. PSO-SVR technique is utilized to predict the boost converter reference current at different irradiation levels so as to extract maximum power from the PV module. The methodology adopted in the research work combined both SPO and SVR 
to tune the SVR parameters. This approach not only improved the accuracy but reduced the number of iterations also since SVR estimation error severely depends on the tuning of its parameters. In the proposed method, the SVR parameters estimated first are used in the offline training stage along with the training data to map the input-output relationship.

The PSO-SVR method is found to exhibit high estimation accuracy and better performance even during rapid fluctuations in the solar irradiation. The tuning of the SVR parameters using PSO method accelerated the estimation process and gave high estimation accuracy to the SVR. Moreover, the drawbacks while using SVR's random parameters have been eliminated, which led to the improvement in the steady state and transient responses of the MPPT controller at different irradiation levels. The experimental results have confirmed the viability, effectiveness, fast transient performance, and high MPPT efficiency for different irradiation level.

\section{ACKNOWLEDGMENT}

The author would like to thank the Deanship of Scientific Research at Majmaah University for supporting this work under Project No. 1441/19.

\section{REFERENCES}

Abo-khalil, A.G. Lee, D.C. Choi, J.W. \& Heung-Geun Kim H.G. 2006. Maximum Power Point Tracking Controller Connecting PV System to Grid. Journal of Power Electronics, 6(3): 226-34.

Abo-khalil, A.G. \& Lee, D.C. 2007. SVR-based wind speed estimation for power control of wind energy generation system. Power Conversion Conference-Nagoya, Japan: 1431-36.

Abo-khalil, A.G. \& Lee, D.C. 2008. MPPT control of wind generation systems based on estimated wind speed using SVR. IEEE Transaction on Industrial Applications, 55(3): 1489-90.

Abo-khalil, A.G. \& Lee, D.C. 2008. Optimal efficiency control of induction generators in wind energy conversion systems using support vector regression. Journal of Power Electronics, 8(4): 345-53.

Abo-Khalil, A.G. 2011. Gradient Approximation Based Maximum Power Point Tracking for PV Grid Connected System. The Power Electronics and Drive Systems Conference PEDS, Singapore.

Abo-Khalil, A.G. \& Abo-Zied, H. 2012. Sensorless control for dfig wind turbines based on support vector regression. In IECON 2012-38th Annual Conference on IEEE Industrial Electronics Society: 3475-80.

Abo-khalil, A.G. Awan, A.B. \& Shaftichit, T. 2016. Feasibility and Estimation of Technical Potential and Calculation of Payback Period of Roof-Top Solar PV System in the City of Majmaah, Province of Riyadh, K.S.A. Journal of Energy and Natural Resources, 5(1): 12-8.

Abo-Khalil, A.G. Alyami, S. Sayed, K. \& Elhejji A. 2019. Dynamic Modeling of Wind Turbines Based on Estimated Wind Speed under Turbulent Conditions. Energies, 12(10): 1-25.

Al-Saleh, Y.M. 2007. A glimpse into the status and prospect of renewables in oil-producing countries; with a special reference to the Kingdom of Saudi Arabia. Geopolitics of Energy, 29(11): 2-13.

Al-Amoudi A. \& Zhang L. 2000. Application of Radial Basis Function Networks for Solar-Array Modeling and Maximum PowerPoint Prediction. IEE Proc. on Generation, Transmission and Distribution, 147(5): 310-16.

Awan, A.B. Zubair, M. Praveen, R.P. \& Abokhalil, A.G. 2018. Solar Energy Resource Analysis and Evaluation of Photovoltaic System Performance in Various Regions of Saudi Arabia. Sustainability, 10: 1129-40.

Cherkassky, V. \& Mulier F. 1998. Learning from Data Concepts, Theory, and Methods. Hoboken, NJ: Wiley.

Cortes, C. \& Vapnic. V. N. 1995. Support-Vector Networks. Machine learning, 20(3): 273-97.

Elgendy, M.A. Zahawi, B. \& Atkinson, D.J. 2012. Assessment of perturb and observe MPPT algorithm implementation techniques for PV pumping applications. IEEE Transaction on Sustainable Energy, 3: 21-33.

Eltamaly, A.M. Al-Saud, M.S. \& Abo-Khalil, A.G. 2020. Photovoltaic maximum power point tracking under dynamic partial shading changes by novel adaptive particle swarm optimization strategy. Transactions of the Institute of Measurement and Control, 42(1): 104-115. 
Farayola, A.M. Hasan, A.N. \& Ali, A. 2017. Implementation of modified incremental conductance and fuzzy logic MPPT techniques using MCUK converter under various environmental conditions. Applied Solar Energy, 53: 173-184.

Femia, N. Petrone, G. Spagnuolo, G. \& Vitelli, M. 2005. Optimization of perturb and observe maximum power point tracking method. IEEE Transaction on Power Electronics, 20(4): 963-73.

Hohm D.P. \& Ropp M.E. 2003. Comparative study of maximum power point tracking algorithms. Progress in Photovoltaics: Research and Applications, 11(1): 47-62.

Hussein, K.H. Muta, I. Hoshino, T. \& Osakada M. 1995. Maximum Photovoltaic Power Tracking: an Algorithm for Rapidly Changing Atmospheric Conditions. IEE Proceeding on Generation, Transmission, and Distribution,142(1): 59-64.

Hsiao, P.W. Chang, C.H. \& Tsai, H.L. 2010. Accuracy improvement of practical PV model. In Proceedings of the SICE Annual Conference, Taiwan: 2725-30.

Jain S. \& Agarwal, V. 2007. Comparison of the performance of maximum power point tracking schemes applied to single-stage grid-connected photovoltaic systems. IET Electric Power Applications, 1(5): 753-62.

Karami, N. Moubayed, N. \& Outbib, R. 2017. General review and classification of different MPPT Techniques. Renewable and sustainable Energy Reviews. 68: 1-18.

Kennedy, J. \& Eberhart R. 1995. Particle swarm optimization. International Conference on Neural Networks (ICNN'95), Australia: 1942-48.

King Abdullah Petroleum Studies and Research Center (KAPSRC). 2017. Growth Through Diversification and Energy Efficiency: Energy Productivity in Saudi Arabia. Consultation Report.

Kottas, T.L. Boutalis, Y.S. \& Karlis, A.D. 2006. New maximum power point tracker for PV arrays using fuzzy controller in close cooperation with fuzzy cognitive networks. IEEE Transaction on Energy Conversion, 21: 793-803.

Lacerda, V.S. Barbosa, P.G. \& Braga, H.A.C. 2010. A single-phase single-stage high power factor grid-connected PV system, with maximum power point tracking. in IEEE Int. Conf. on Industrial Technology (ICIT) : 871-77.

Lin, S.W. Ying, K.C. Chen, S.C. \& Lee Z.J. 2008. Particle swarm optimization for parameter determination and feature selection of support vector machines. Expert System Applications, 35(4): 1817-24.

Mohanty, S. Subudhi, B. \& Ray, P.K. 2017. A Grey Wolf-Assisted Perturb \& Observe MPPT Algorithm for a PV System. IEEE Transaction on Energy Conversion, 32: 340-47.

Muller, K.R. Smola, A. Ratsch, G. Scholkopf, B. Kohlmorgen, J. \& Vapnik, V. 1997. Predicting time series with support vector machines. in Proc. ICANN. New York: Springer-Verlag, 1327: 999-1004.

Piegari L. \& Rizzo, R. 2010. Adaptive perturb and observe algorithm for photovoltaic maximum power point tracking. IET Renewable Power Generation, 4(4): 317-28.

Safari, A. \& Mekhilef, S. 2011. Incremental conductance MPPT method for PV systems. In Proceedings of the 24th Canadian Conference on IEEE, Niagara Falls, ON, Canada, 345-47.

Spiazzi, G. Buso, S. \& Mattavelli P. 2009. Analysis of MPPT algorithms for photovoltaic panels based on ripple correlation techniques in presence of parasitic components. in 2009 Brazilian Power Electronics Conference, COBEP2009: 88-95.

Syafaruddin, Karatepe, E. \& Hiyama, T. 2009. Artificial neural network-polar coordinated fuzzy controller based maximum power point tracking control under partially shaded conditions, IET Renewable Power Generation, 3(2): 239-253.

Vapnic. V.N. 1999. An overview of statistical learning theory. IEEE Transaction on Neural Network, 10(5): 988-99.

Veerachary, M. Senjyu, T. \& Uezato, K. 2003. Neural-network-based maximum-power-point tracking of coupled-inductor interleaved-boost converter- supplied PV system using fuzzy controller. IEEE Transaction Industrial Electronics, 50(4): 749-58.

Yu, B.G. Abo-khalil, A.G. Matsui, M. \& Yu, G.J. 2009. Sensorless Fuzzy Logic Controller for Maximum Power point Tracking of Grid Connected PV System. The International Conference of Electrical Machines and Systems (ICEMS), 1: 5-9.

Yu, B.G. Matsui, M. Abo-Khalil, A.G. \& Yu, G. 2009. A Correlation-Based Islanding Detection Method Using Current Disturbance for PV System. In Proceedings of the International Conference on Electrical Machines and Systems ICEMS, Japan, 15-18.

Zell, Z. Gasim, S. Wilcox, S. Katamoura, S. Stoffel, T. Shibli, S. Engel-Cox, J. \& AlSubie M. 2015. Assessment of solar radiation resources in Saudi Arabia. Solar Energy, 119: 422-38. 